\title{
EFEITO DA DESFOLHA NA FASE VEGETATIVA EM ALGUNS CARACTERES AGRONÔMICOS DA CULTIVAR DE SOJA BMX POTÊNCIA RR
}

Carlos André Bahry ${ }^{1}$, Edmar Lopes Dantas ${ }^{2}$, Eduardo Venske ${ }^{1}$, Maicon Nardino ${ }^{3}$, Paulo Dejalma Zimmer $^{1}$, Velci Queiróz de Souza ${ }^{3}$, Braulio Otomar Caron ${ }^{3}$

${ }^{1}$ Universidade Federal de Pelotas, Faculdade de Agronomia Eliseu Maciel, Rio Grande do Sul, E-mail: carlosbahry@ hotmail.com

${ }^{2}$ Empresa Agropastoril Jotabasso LTDA. Ponta Porã, Mato Grosso do Sul

${ }^{3}$ Universidade Federal de Santa Maria, Campus de Frederico Westphalen, Rio Grande do Sul

\section{RESUMO}

O objetivo do trabalho foi avaliar o efeito da desfolha na fase vegetativa em alguns caracteres agronômicos da soja. A cultivar utilizada foi a BMX Potência RR. Os tratamentos foram: T1: sem desfolha; T2: em V4, remoção do par de folhas unifolioladas; T3: em V4, remoção do par de folhas unifolioladas e em V5, do $1^{\circ}$ trifólio; T4: em V4, remoção do par de folhas unifolioladas; V5, $1^{\circ}$ trifólio; V6, $2^{\circ}$ trifólio; T5: em V4, remoção do par de folhas unifolioladas; V5, $1^{\circ}$ trifólio; V6, $2^{\circ}$ trifólio; V7, $3^{\circ}$ trifólio; T6: em V4 remoção do par de folhas unifolioladas; V5, $1^{\circ}$ trifólio; V6, $2^{\circ}$ trifólio; V7, $3^{\circ}$ trifólio; V8, $4^{\circ}$ trifólio; T7: em V4, remoção do par de folhas unifolioladas; V5, $1^{\circ}$ trifólio; V6, $2^{\circ}$ trifólio; V7, $3^{\circ}$ trifólio; V8, $4^{\circ}$ trifólio; V9, $5^{\circ}$ trifólio. O delineamento foi em blocos ao acaso, com quatro repetições. Analisou-se: altura de inserção do primeiro legume, altura da haste principal, índice de colheita, massa de mil sementes e produtividade. Após análise de variância, as médias foram comparadas pelo Teste de Tukey a $5 \%$ de probabilidade. A desfolha na fase vegetativa não afetou a altura da haste principal e o índice de colheita. A altura de inserção do primeiro legume, a produtividade e a massa de mil sementes foram afetadas negativamente quando mais estádios vegetativos foram submetidos à desfolha.

Palavras-chave: produtividade, massa de mil sementes, índice de colheita

\section{EFFECT OF DEFOLIATION ON THE VEGETATIVE PHASE IN SOME AGRONOMIC FEATURES OF BMX POTÊNCIA RR SOYBEAN CULTIVAR}

\section{ABSTRACT}

The aim of this work was to evaluate the effect of defoliation on the vegetative phase in some agronomic characters of soybean. The cultivar used was BMX Potência RR. The treatments were: T1: without defoliation; T2: in V4, removal of the unifoliolate leaves pair; T3: in V4, removal of the unifoliolate leaves pair and in V5, of the first trefoil; T4: in V4, removal of the unifoliolate leaves pair; in V5, of the first trefoil; in V6, second trefoil; T5: in V4, removal of the unifoliolate leaves pair; in V5, the first trefoil; in V6, second trefoil; in V7 third trefoil; T6: in $\mathrm{V} 4$, removal of the unifoliolate leaves pair; in V5, the first trefoil; in V6, second trefoil; in V7 third trefoil; in V8, fourth trefoil; T7: in V4, removal of the unifoliolate leaves pair; in V5, the first trefoil; in V6, second trefoil; in V7 third trefoil; in V8, fourth trefoil; in V9, fifth trefoil. The design was in randomized block, with four replicates. It was analyzed: height of first pod, height of main branch, harvest index, weight of thousand seeds and yield. After variance analysis, the media were compared by Tukey Test at $5 \%$ probability. The defoliation in the vegetative phase did not affect the height of main branch and the harvest index. The height of first pod, yield and weight of thousand seeds were negatively affected when more vegetative stages were subjected to defoliation.

Keywords: yield, thousand seeds mass, harvest index 


\section{INTRODUÇÃO}

A área foliar e a sua capacidade fotossintética são fatores de importância ímpar na definição do potencial produtivo da soja, pois é a partir das folhas e de sua interação com a radiação solar que os fotoassimilados são produzidos e destinados para o enchimento de grãos (ANDRADE $e t$ $a l .$, 2002). Dessa maneira, qualquer fator que limite a área foliar irá comprometer a fotossíntese e, consequentemente, a produtividade final da cultura.

Diversos são os fatores que podem comprometer o adequado processo fotossintético, dentre os quais a baixa radiação incidente sobre o dossel, o ataque de insetos desfolhadores, doenças como a ferrugem da soja, que provoca queda precoce das folhas, estresses abióticos, como a seca, a salinidade e a fertilidade do solo (PEDIGO et al., 1986; YANG et al., 2001; LI et al., 2006; TIMSINA et al., 2007; DEBONA et al., 2009).

O potencial de recuperação da soja quando submetida a uma desfolha depende muito de suas características genéticas, do estádio de desenvolvimento em que se encontram as plantas, e da própria intensidade do desfolhamento (COSTA et al., 2003).

Pesquisas a respeito do tema têm mostrado que desfolhas inferiores a 50\%, antes do florescimento, normalmente não reduzem a produtividade da soja. No entanto, quando ocorre na fase reprodutiva, as perdas podem chegar a $87 \%$ (PRATISSOLI et al., 2001; PARCIANELLO et al., 2004; PELUZIO et al., 2004; FONTOURA et al., 2006).

Baseado na importância da manutenção foliar da soja, o presente trabalho teve como objetivo avaliar o efeito da desfolha na fase vegetativa em alguns caracteres agronômicos da cultivar de soja BMX Potência RR.

\section{MATERIAL E MÉTODOS}

O trabalho foi conduzido durante o ano agrícola 2010/2011, na área de produção de sementes da Empresa Agropastoril Jotabasso Ltda., situada no município de Ponta Porã, Mato Grosso do Sul (latitude de $22^{\circ} 16^{\prime} 07^{\prime \prime}$ Sul, longitude de $55^{\circ} 32^{\prime} 12^{\prime \prime}$ Oeste, e altitude de 538 metros).

A cultivar de soja utilizada foi a BMX Potência RR, de hábito de crescimento indeterminado e grupo de maturação 6.7.

Os tratos culturais seguiram as recomendações técnicas da cultura para a região e a adubação foi realizada de acordo com a análise de solo e o histórico da área, sendo aplicados $300 \mathrm{~kg} \mathrm{ha}^{-1}$ da fórmula NPK 00-20-20 + micronutrientes.

A demarcação do local do experimento foi realizada após a emergência das plântulas de soja. O estande final obtido foi de 12 plantas $\mathrm{m}^{-1}$. Cada unidade experimental foi constituída de 4 linhas de 3 metros de comprimento, com $0,45 \mathrm{~m}$ entre linhas, totalizando uma área total de $5,4 \mathrm{~m}^{2}$.

A metodologia constou de sete tratamentos: tratamento 1: controle, sem desfolha; tratamento 2: em V4 retirada do par de folhas unifolioladas; tratamento 3: em V4 retirada do par de folhas unifolioladas, em V5 retirada do primeiro trifólio; tratamento 4: em V4 retirada do par de folhas unifolioladas, em V5 retirada do primeiro trifólio, em V6 retirada do segundo trifólio; tratamento 5: em V4 retirada do par de folhas unifolioladas, em V5 retirada do primeiro trifólio, em V6 retirada do segundo trifólio, em V7 retirada do terceiro trifólio; tratamento 6: em V4 retirada do par de folhas unifolioladas, em V5 retirada do primeiro trifólio, em V6 retirada do segundo trifólio, em V7 retirada do terceiro trifólio, em V8 retirada do quarto trifólio; tratamento 7: em V4 retirada do par de folhas unifolioladas, em V5 retirada do primeiro trifólio, em V6 retirada do segundo trifólio, em V7 retirada do terceiro trifólio, em V8 
retirada do quarto trifólio, em V9 retirada do quinto trifólio. A escala fenológica adotada foi a de Fehr \& Caviness (1977).

Semanalmente era realizada uma vistoria no experimento para eliminar as respectivas brotações de cada tratamento. No momento da colheita eliminou-se uma linha de bordadura de cada lado da parcela e $0,5 \mathrm{~m}$ da bordadura de cada linha colhida. As variáveis analisadas foram:

Altura de inserção do primeiro legume: distância entre o nível do solo e a inserção do primeiro legume na haste principal, expresso em centímetros.

Altura da haste principal: distância entre o nível do solo até o ápice da haste principal, expresso em centímetros.

Massa de mil sementes: realizada através da contagem manual de oito repetições de 100 sementes para cada repetição, segundo Brasil (2009), expresso em gramas.

Índice de colheita: massa seca dos grãos, dividida pelo rendimento biológico aparente (massa seca de ramos + massa da haste + massa de legumes com grãos).

Produtividade: massa total das sementes de cada parcela, corrigida para $13 \%$ de umidade, transformando o resultado para hectare e expresso em sacas. Considerou-se uma saca de soja com massa de $60 \mathrm{~kg}$.

$\mathrm{O}$ delineamento experimental foi em blocos ao acaso, com quatro repetições. Realizou-se análise de variância. Para comparação de médias utilizou-se o Teste de Tukey ao nível de 5\% de probabilidade de erro. O programa estatístico utilizado foi o SAS.

\section{RESULTADOS E DISCUSSÃO}

Os resultados obtidos em relação à variável altura de inserção do primeiro legume mostraram que houve diferença significativa entre os tratamentos, tendo o tratamento 7 apresentado maior altura de inserção quando comparado aos tratamentos $1,2,3$ e 4, e não diferindo dos tratamentos 5 e 6 (Tabela 1).

Possivelmente, essa maior altura de inserção do primeiro legume verificada no tratamento 7 esteja diretamente relacionada ao maior número de estádios vegetativos submetidos à desfolha, quando comparado aos demais (Tratamentos 1, 2, 3 e 4). Isso mostra o quão importante é a manutenção das folhas pelo maior tempo possível, em especial as do terço inferior, pois com sua queda precoce, aqui representada pela desfolha artificial, mas apoiada em um ataque de insetos desfolhadores, incidência precoce da ferrugem da soja, como ocorre no cerrado, estresses abióticos diversos (YANG et al., 2001; LI et al., 2006; TIMSINA et al., 2007; DEBONA et al., 2009), mais alta será a inserção dos legumes na haste principal, potencializando a redução desse componente de rendimento nas plantas, o que pode comprometer a produtividade final.

Dessa forma, a associação de diversas práticas agrícolas pode contribuir para minimizar os fatores prejudiciais à manutenção das folhas durante a fase vegetativa da soja. Uma semeadura adequada, respeitando a umidade do solo e o zoneamento agroclimático para a região, a adoção do plantio direto, o emprego do manejo integrado de pragas e doenças, uso de irrigação e uma nutrição equilibrada das plantas, são medidas importantes a serem consideradas pelos agricultores, visando o estabelecimento e o desenvolvimento satisfatório das plantas na lavoura.

A altura da haste principal não apresentou diferença em função dos diferentes tratamentos aplicados nesse estudo (Tabela 1). Esse resultado não corrobora com os verificados por Peluzio et al. (2004), os quais observaram redução na altura das plantas à medida que se aumentou a desfolha da soja.

$\mathrm{O}$ índice de colheita apresentou diferença significativa entre os tratamentos, 
apresentando os tratamentos 2 e 5 , valores inferiores aos tratamentos 4, 6 e 7, mas não diferindo dos demais. Esses valores de índice de colheita estão apropriados para a cultura da soja (Tabela 1). No entanto, não foram encontrados relatos na literatura relacionando $\mathrm{o}$ índice de colheita e a desfolha precoce na fase vegetativa da soja, mas observou-se, no presente estudo, que essa variável não sofreu influência da desfolha. Os tratamentos que tiveram mais estádios vegetativos submetidos à desfolha, nesse caso o 6 e o 7, não diferiram por exemplo, do tratamento 1 , sem aplicação de desfolha.

Para a variável massa de mil sementes também se verificou diferença significativa entre os tratamentos, tendo apresentado o tratamento 7 a menor massa dentre os demais tratamentos avaliados (Tabela 1). Esse fato pode estar relacionado à limitação de área foliar das plantas em função da desfolha, logo, menor produção de fotoassimilados para o enchimento de grãos. Esse resultado corrobora com os encontrados por Peluzio et al. (2004). Porém, segundo Fontoura et al. (2006), a massa de mil sementes somente foi afetada quando as plantas foram submetidas à desfolha na fase reprodutiva e principalmente, quando a intensidade foi de $100 \%$.

A soja tolera certo índice de desfolha proporcionando às folhas remanescentes maior eficiência na taxa fotossintética quando comparado às plantas sem desfolha, em especial quando submetidas a algum estresse abiótico (LI et al., 2006). Porém, apesar da taxa fotossintética não ter sido avaliada no presente estudo, acredita-se que, mesmo tendo havido maior eficiência das folhas remanescentes do tratamento 7 , essas não conseguiram compensar toda a demanda dos drenos, nesse caso os grãos, limitando assim a sua massa (Tabela 1).

Essa simulação de desfolha permanente é semelhante ao ataque severo de ferrugem da soja, a qual traz como prejuízo principal a queda prematura das folhas e, consequentemente, a redução da massa de grãos, pela redução na fonte, prejudicando, dessa forma a produtividade da cultura (YANG et al., 2001). Situação semelhante é verificada quando do ataque de insetos desfolhadores onde, havendo a limitação de área foliar pode ocorrer menor produção de fotoassimilados, comprometendo o adequado enchimento dos grãos (TIMSINA et al., 2007).

A produtividade seguiu a tendência da massa de mil sementes, onde à medida que se aumentou os níveis de desfolha foi possível visualizar uma redução na produtividade da cultura (Tabela 1; Figura 1), em especial no tratamento 7. Essa diferença chegou a 7,4 sacas de soja por hectare em relação ao tratamento 1 , sem desfolha, o que é um valor considerável em se tratando de uma cultura que normalmente é cultivada em grandes áreas. Isso representou, ainda na comparação desses dois tratamentos, uma redução de $11,85 \%$ na produtividade.

Diversos autores apontam a desfolha precoce, em especial na fase reprodutiva da cultura, como um fator importante e que pode limitar a produtividade final (PRATISSOLI et al., 2001; YANG et al., 2001; PARCIANELLO et al., 2004; PELUZIO et al., 2004; FONTOURA et al., 2006).

Apesar da comprovação de que o cuidado com a desfolha severa na fase vegetativa é imprescindível, seja ela devido ao ataque de pragas, doenças, fertilidade do solo, estresses abióticos diversos, como estiagem ou salinidade, verificou-se que a média de produtividade de todos os tratamentos juntos, 59,5 sacas por ha, ficou acima da média nacional, que é de 47 sacas por ha (CONAB, 2010). 
Tabela 1. Análise das variáveis: altura de inserção do primeiro legume (IPL); altura da haste principal (AHP); índice de colheita (IC); massa de mil sementes (MMS); e produtividade, em sacas por ha em função dos tratamentos de desfolha aplicados em diferentes estádios vegetativos da cultivar de soja BMX Potência RR.

\begin{tabular}{cllccc}
\hline Tratamento & $\begin{array}{l}\text { IPL } \\
(\mathrm{cm})\end{array}$ & $\begin{array}{c}\text { AHP } \\
(\mathrm{cm})\end{array}$ & IC & $\begin{array}{c}\text { MMS } \\
(\mathrm{g})\end{array}$ & $\begin{array}{c}\text { Produtividade }^{-1} \\
\text { sacas.ha }^{-1}\end{array}$ \\
\hline 1 & $12,0 \mathrm{c}^{*}$ & $79,5 \mathrm{a}$ & $0,54 \mathrm{ab}$ & $152,0 \mathrm{a}$ & $62,4 \mathrm{a}$ \\
2 & $13,0 \mathrm{bc}$ & $80,3 \mathrm{a}$ & $0,52 \mathrm{~b}$ & $149,0 \mathrm{a}$ & $61,0 \mathrm{a}$ \\
3 & $12,5 \mathrm{c}$ & $79,5 \mathrm{a}$ & $0,55 \mathrm{ab}$ & $151,0 \mathrm{a}$ & $60,8 \mathrm{a}$ \\
4 & $13,3 \mathrm{bc}$ & $79,0 \mathrm{a}$ & $0,56 \mathrm{a}$ & $148,0 \mathrm{a}$ & $59,9 \mathrm{a}$ \\
5 & $14,0 \mathrm{abc}$ & $80,0 \mathrm{a}$ & $0,53 \mathrm{~b}$ & $149,0 \mathrm{a}$ & $58,9 \mathrm{ab}$ \\
6 & $15,0 \mathrm{ab}$ & $77,0 \mathrm{a}$ & $0,58 \mathrm{a}$ & $146,0 \mathrm{a}$ & $58,6 \mathrm{ab}$ \\
7 & $15,8 \mathrm{a}$ & $78,0 \mathrm{a}$ & $0,58 \mathrm{a}$ & $123,0 \mathrm{~b}$ & $55,0 \mathrm{~b}$ \\
\hline $\mathrm{CV}(\%)$ & 6,9 & 1,8 & 2,9 & 1,9 & 3,0 \\
\hline
\end{tabular}

*médias seguidas pela mesma letra na coluna não diferem entre si pelo Teste de Tukey ao nível de 5\% de probabilidade de erro.

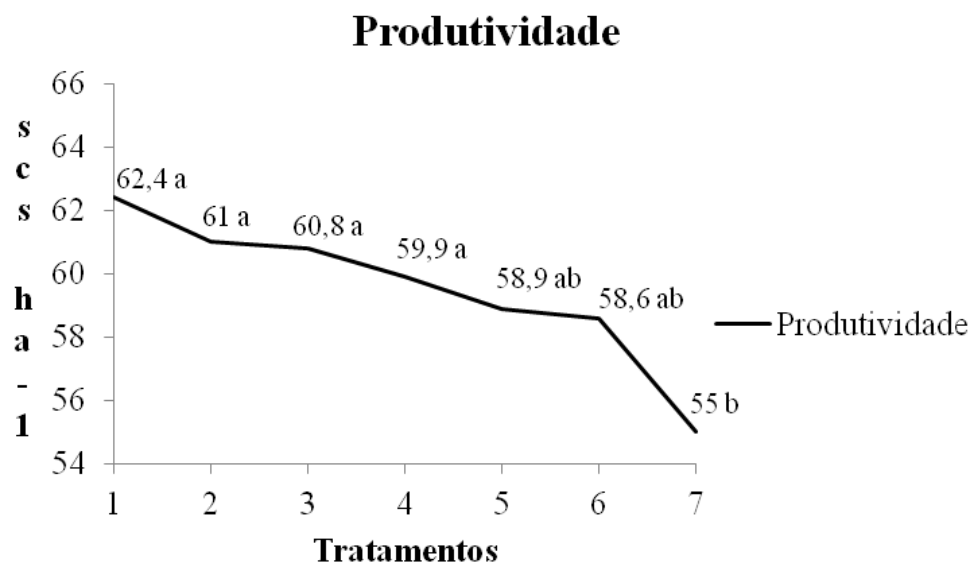

Figura 1. Dados médios de produtividade em função dos tratamentos de desfolha aplicados em diferentes estádios vegetativos da cultivar de soja BMX Potência RR.

No entanto, considerando o tratamento 1 e o tratamento 7 , esse último apresentou uma proximidade maior da média nacional, com 55 sacas por ha, tendo o tratamento sem desfolha, alcançado uma produtividade média de 62,4 sacas por ha.

\section{CONCLUSÕES}

A desfolha na fase vegetativa não afetou a altura da haste principal e o índice de colheita.

A altura de inserção do primeiro legume, a produtividade e a massa de mil sementes foram afetadas negativamente quando mais estádios vegetativos foram submetidos à desfolha.

\section{REFERÊNCIAS BIBLIOGRÁFICAS}

ANDRADE, F.H.; CALVIÑO, P.; CIRILO, A.; BARBIERI, P. 2002. Yield Responses to Narrow Rows Depend on Increased Radiation Interception. Agronomy Journal, Madison, v.94, n.5, p.975-980.

BRASIL. 2009. Regras para análise de sementes. Ministério da Agricultura, Pecuária e Abastecimento. Brasília, DF, ed. $1^{\mathrm{a}}, 398 \mathrm{p}$. 
CONAB. 2010. Safras: comparativo de área, produção e produtividade. Disponível em: <http://www.conab.gov.br>. Acesso em: 15 de maio de 2012.

COSTA, M.A.G.; BALARDIN, R.S.; COSTA, E.C.; GRÜTZMACHER, A.D.; SILVA, M.T.B. 2003. Níveis de desfolha na fase reprodutiva da soja, cv Ocepar 14, sobre dois sistemas de cultivo. Ciência Rural, Santa Maria, v.33, n.5, p.813-819.

DEBONA, D.; FIGUEIRÓ, G.G.; CORTE, G.D.; NAVARINI, L.; DOMINGUES, L. da S. BALARDIN, R.S. 2009. Efeito do tratamento de sementes com fungicidas e acibenzolar-S-methyl no controle da ferrugem asiática e crescimento de plântulas em cultivares de soja. Summa Phytopathology, v.35, n.1, p.26-31.

FEHR, W.R.; CAVINESS, C.E. 1977. Stages of soybean development. Ames: State University of Science and Technology. 11 p. (Special report, 80).

FONTOURA, T.B.; COSTA, J.A.; DAROS, E. 2006. Efeito de níveis e épocas de desfolhamento sobre o rendimento e os componentes do rendimento de grãos da soja. Scientia Agraria, v.7, n.1-2, p.4954.

LI, X.; AN, S.; ENEJI, A.E.; TANABE, K. 2006. Salinity and defoliation effects on soybean growth. Journal of Plant Nutrition. v.29, n.8, p.1499-1508.

PARCIANELLO, G.; COSTA, J.A.; PIRES, J.L.F.; RAMBO, L.; SAGGIN, K. 2004. Tolerância da soja ao desfolhamento afetada pela redução do espaçamento entre fileiras. Ciência Rural, Santa Maria, v.34, n.2, p.357-364.

PEDIGO, L.P.; HUTCHINS, S.H.; HIGLEY, L.G. 1986. Economic injury levels in theory and practice. Annual Review of Entomology, Palo Alto, v.31, p.341-368.
PELUZIO, J.M.; BARROS, H.B.; BRITO, E.L.; SANTOS, M.M. dos; SILVA, R.R. da. 2004. Efeitos sobre a soja do desfolhamento em diferentes estádios fenológicos. Revista Ceres, Viçosa, v.51, n.297, p.575-585.

PRATISSOLI, D.; SCHMILDT, E.R.; REIS, E.F.; THULER, R.T. 2001. Influência de desfolhas simuladas na produtividade e em outras características agronômicas do feijoeiro. Revista Ceres, Viçosa, v.48, n. 275, p.17-24.

TIMSINA, J.; BOOTE, K.J.; DUFFIELD, S. 2007. Evaluating the CROPGRO soybean model for predicting impacts of insect defoliation and depodding. Agronomy Journal, v.99, n.1, p.148157.

YANG, X.B.; TSCHANZ, A.T.; DOWLER, W.M.; WANG, T.C. 2001. Development of yield loss models in relation to reductions of components of soybeans infected with Phakopsora pachyrhizi. Phytopathology, St. Paul, v.81, p.14201426.

Recebido em: 4/06/2012 Aceito para publicação em: 27/11/2013 